\title{
Size does matter: Nanobodies as a new format for tumor imaging probes.
}

\author{
Marta M Kijanka \\ Senior Scientist, Vice President, R\&D, Utrechtseweg, Amersfoort, Netherlands.
}

\section{Editorial}

Currently there are several imaging techniques that enable detection and staging of cancer, namely X-ray, computed tomography (CT), ultrasonography (US), magnetic resonance imaging (MRI), single photon emission computed tomography (SPECT), positron emission tomography (PET) and optical imaging [1]. Each of these modalities comes with a set of advantages and limitations. Increasing understanding of cancer biology has led in the recent years to determination of several cancer biomarkers. This knowledge on molecules which expression is upregulated in cancer cells in comparison to normal healthy cells allowed development of targeted pharmaceuticals. Improving tumor specificity of used pharmaceuticals was the key step leading to high contrast imaging. Firstly, monoclonal antibodies were used as imaging agents. To this day 5 monoclonal antibodies are approved by FDA for diagnostic imaging [2].

Which properties should have an ideal imaging probe? Providing specific recognition of cancer cells due to high affinity to targets expressed, ideally, exclusively on the surface of these cells is the first requirement. Secondly, rapid extravasation from systemic circulation into the tumor site and its homogenous penetration is essential. Due to this sufficient accumulation at the tumor site may be obtained resulting in high specific signal already shortly upon probe injection. Finally, it should be efficiently removed from non-targeted tissues, which translate to quality of contrast, and non-immunogenic to facilitate multiple administrations during the course of treatment monitoring.

Monoclonal antibodies are rather large molecules composed of 2 light chains and 2 heavy chains. They are known for their long circulation upon intravenous injection, which is beneficial when used as therapeutics, however undesired when considering them as tumor imaging tools. Their prolonged presence in circulation may last from a few days to weeks, resulting in obtaining satisfactory contrast between tumor and normal tissue not earlier than 2 to 4 days post injection.
It became clear that conventional antibodies, mostly due to their large size, do not fulfil these requirements. Over the years, a lot of research was conducted aiming at finding the ultimate format for an imaging probe. When reviewing the available literature we are witnessing down-sizing from monoclonal antibodies $(150 \mathrm{kDa})$, through single chain variable fragments $(30 \mathrm{kDa})$, to as small as antibodies $(7 \mathrm{kDa})$ [3-5]. However, what needs to be kept in mind is that the advantage of smaller size is counterbalanced by either decreased stability or lack of sufficiently high affinity towards the target [6]. Is there then a good alternative to monoclonal antibodies as imaging probes?

In the early 1990s a new type of antibodies - the heavy chain antibodies (HcAb, $95 \mathrm{kDa}$ ) was discovered by HamersCasterman et al. [7]. These fully functional structures found in Camelidae and in cartilaginous fish are homodimers of two heavy chains, with $\mathrm{CH} 2, \mathrm{CH} 3$ and variable domains, connected by disulphide bonds, but they lack a light chain. Interaction of these molecules with appropriate antigens is mediated by amino acids residing in 3 complementaritydetermining regions (conventional $\mathrm{mAb}$ contains $6 \mathrm{CDR}$ loops, 3 present in each heavy chain and 3 present in each light chain) of the single variable domain (referred to as VHH or nanobody). The CDR loops are extended in comparison to loops present in conventional $\mathrm{Ab}$ (ranging from 8 to 24 residues, while in conventional human $\mathrm{mAb}$ they are usually 12 amino acids long). This extension of length provides large enough antigen interacting surface [8]. Nanobodies, 10 times smaller in molecular weight that conventional $\mathrm{mAbs}$ are fully functional, smallest naturally derived antigen binding fragments.

Recently nanobodies have attracted much attention as they fulfil all requirements for an ideal imaging probe. They possess high affinity towards their targets. Due to their small size, they rapidly extravasate from the bloodstream and penetrate tumors in a homogenous manner. Furthermore, they are described as having low immunogenic potential due to high similarity to human $\mathrm{VH}$ domains, high stability and rapid clearance from blood. Unbound nanobodies are cleared fast, allowing acquiring of images of high contrast between tumor and healthy tissue shortly after their administration. 
So far nanobodies have been tested in preclinical studies as imaging probes suitable for nuclear imaging, optical imaging and ultrasound. Findings of these studies are well described in a review by Oliveira et al. [9]. Having in mind positive evaluation of nanobodies as imaging probes suitable for the above mentioned modalities, imagining nanobodies entering daily clinical practice does not seem to be just wishful thinking anymore. Nanobody based imaging probes could be employed not just in tumor detection or confirmation of target expression prior to start of treatment, but also to follow response to the treatment (allowing early identification of non-responding subset of patients) or detection of possible tumor re-occurence. Furthermore, due to their fast accumulation at the tumor site nanobodies could be used as tools in image-guided surgery-enabling surgeons to remove the entire tumor, while preserving healthy surrounding tissues.

Earlier this year Keyaerts et al. published results on the first-in-human application of a ${ }^{68} \mathrm{Ga}$ radiolabeled HER2 specific nanobody for PET imaging [10]. 20 women with primary or metastatic breast carcinoma (score of $2^{+}$ or $3^{+}$on HER2 immunohistochemical assessment) were included in the described study. No adverse reactions were observed. The tracer showed fast blood clearance, with only $10 \%$ of injected activity remaining in the blood at $1 \mathrm{~h}$ after injection. Keyaerts et al. [10] reported highest probe uptake to be in the kidneys, liver, and intestines but very low background levels in all other organs. Tracer accumulation in tumor lesions could be evaluated in 19 patients (in 13 of 15 primary tumors), while all patients with metastatic lesions showed clear tracer accumulation in at least 1 lesion. Finally, ${ }^{68} \mathrm{Ga}$-HER2-Nanobody PET/ CT was shown to be a safe procedure with a radiation dose comparable to that of other routinely used PET tracers.

In conclusion, obtained results justified further assessment of this nanobody-based tracer in a phase II trial. With this study we might be witnessing the blooming of a new era of nanobody-based tracers for cancer imaging.

\section{References}

1. Belkic D, Belkic K. Molecular imaging in the framework of personalized medicine. Isr Med Assoc J 2013; 15: 665-672.

2. van Dongen GA, Visser GW, Lub-de Hooge $\mathrm{MN}$, et al. Immuno-PET: A navigator in monoclonal antibody development and applications. Oncologist 2007; 12: 13791389 .

3. Zielinski R, Hassan M, Lyakhov I, et al. Affibody-DyLight conjugates for in vivo assessment of HER2 expression by near-infrared optical imaging. PLoS One 2012; 7: e41016.

4. Zdobnova TA, Stremovskiy OA, Lebedenko EN, et al. Selfassembling complexes of quantum dots and $\mathrm{scFv}$ antibodies for cancer cell targeting and imaging. PLoS One 2012; 7: e48248.

5. Kaur S, Venktaraman G, Jain M, et al. Recent trends in antibody based oncologic imaging. Cancer Lett 2012; 315: 97-111.

6. Bell A, Wang ZJ, Arbabi-Ghahroudi M, et al. Differential tumor-targeting abilities of three single domain antibody formats. Cancer Lett 2010; 289: 81-90.

7. Hamers-Casterman C, Atarhouch T, Muyldermans S, et al. Naturally occurring antibodies devoid of light chains. Nature 1993; 363: 446-448.

8. Bruce VJ, Ta AN, McNaughton BR. Minimalist antibodies and mimetics: An update and recent applications. Chembiochem 2016; 17: 1892-1899.

9. Oliveira S1, Heukers R, Sornkom J, et al. Targeting tumors with nanobodies for cancer imaging and therapy. J Control Release 2013; 172: 607-617.

10. Keyaerts M, Xavier C, Heemskerk J, et al. Phase I Study of ${ }^{68} \mathrm{Ga}$-HER2-Nanobody for PET/CT assessment of HER2 expression in breast carcinoma. J Nucl Med 2016; 57: 27-33.

\section{Correspondence to:}

Marta M Kijanka,

Senior Scientist, Vice President R\&D,

Utrechtseweg 223,

3818 EE Amersfoort,

Netherlands.

E-mail: kijanka.marta@gmail.com 